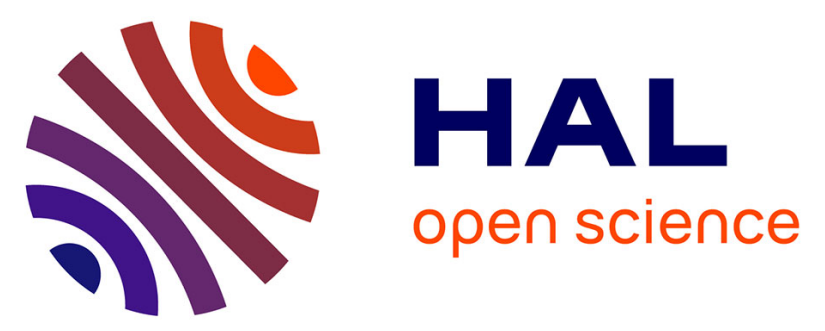

\title{
Shifted Legendre polynomials algorithm used for the dynamic analysis of PMMA viscoelastic beam with an improved fractional model
}

Jiawei Cao, Yiming Chen, Yuanhui Wang, Gang Cheng, Thierry Barriere

\section{- To cite this version:}

Jiawei Cao, Yiming Chen, Yuanhui Wang, Gang Cheng, Thierry Barriere. Shifted Legendre polynomials algorithm used for the dynamic analysis of PMMA viscoelastic beam with an improved fractional model. Chaos, Solitons \& Fractals, 2020, 141, pp.110342 (9). hal-03020551

\section{HAL Id: hal-03020551 https://hal.science/hal-03020551}

Submitted on 23 Nov 2020

HAL is a multi-disciplinary open access archive for the deposit and dissemination of scientific research documents, whether they are published or not. The documents may come from teaching and research institutions in France or abroad, or from public or private research centers.
L'archive ouverte pluridisciplinaire HAL, est destinée au dépôt et à la diffusion de documents scientifiques de niveau recherche, publiés ou non, émanant des établissements d'enseignement et de recherche français ou étrangers, des laboratoires publics ou privés. 
Elsevier Editorial System(tm) for Chaos,

Solitons \& Fractals or its open access mirror

Manuscript Draft

Manuscript Number: CHAOS-D-20-02466R1

Title: Shifted Legendre polynomials algorithm used for the dynamic analysis of PMMA viscoelastic beam with an improved fractional model

Article Type: Full length article

Section/Category: Interdisciplinary applications of Mathematics

Keywords: Fractional calculus, Fractional partial differential equation, Dynamic analysis, Polymethyl methacrylate, Viscoelastic model, Shifted Legendre polynomial

Corresponding Author: Dr. Yiming Chen,

Corresponding Author's Institution:

First Author: Jiawei Cao

Order of Authors: Jiawei Cao; Yiming Chen; Yuanhui Wang; Gang Cheng; Thierry Barriere

Abstract: In this paper, a fractional viscoelastic model is proposed to describe the physical behaviour of polymeric material. The material parameters in the model are characterized by the experimental data obtained in the dynamical mechanical analysis. The proposed model is integrated into the fractional governing equation of polymethyl methacrylate (PMMA) above its glass transition temperature. The numerical algorithm based on the shifted Legendre polynomials is retained to solve the fractional governing equations in the time-domain. The accuracy and effectiveness of the algorithm are verified according to the mathematical examples. The advantage of this method is that Laplace transform and the inverse Laplace transform commonly used in fractional calculus are avoided. The dynamical response of the viscoelastic PMMA beam is determined with several loading conditions (uniformly distributed load and harmonic load). The effects of the loading condition and the temperature on the dynamic response of the beam are investigated in the results. The proposed approach shows great potentials for the highprecision calculation in solving the fractional equations in the science and engineering . 
Dear Prof. Abdon Atangana:

On behalf of my co-authors, we thank you very much for giving us an opportunity to revise our manuscript, we appreciate editor and reviewers very much for their positive and constructive comments and suggestions on our manuscript entitled "Shifted Legendre polynomials algorithm used for the dynamic analysis of PMMA viscoelastic beam with an improved fractional model".(ID:CHAOS-D-20-02466)

We have studied reviewers' comments carefully and tried our best to revise our manuscript according to the comments. In the manuscript the comments for Reviewer 1, Reviewer 2 and Reviewer 4 are all highlighted in blue, red and cyan. We have attached please find the revised version, which we would like to submit for your kind consideration.

We would like to express our great appreciation to you and reviewers for comments on our paper.

Looking forward to hearing from you.

Thank you and best regards.

Yours sincerely,

Cao Jiawei

Co-author:

Name: Yiming Chen

E-mail: chenym@ysu.edu.cn 


\section{Shifted Legendre polynomials algorithm used for the dynamic analysis of PMMA viscoelastic beam with an improved fractional model}

Manuscript ID: CHAOS-D-20-02466

We greatly appreciate the editor's effort in handling our manuscript and the positive and constructive comments from anonymous reviewers. In the revised version, we have addressed all the comments point by point and concerns to our best understanding and capability. The detailed "Response to the reviews" and "Summary of changes" are presented below.

The valuable comments of the reviewers enable us to improve the quality of our manuscript. We believe our response to reviewers will be satisfactory and the revised version is suitable for publication in the prestigious Chaos, Solitons \& Fractals. In the manuscript the comments for Reviewer 1, Reviewer 2 and Reviewer 4 are all highlighted in blue, red and cyan.

\section{Response to the review}

Reviewer 1:

Comment 1: In the abstract line 12, "determinated" need to be changed to "determined."

Response: We are very sorry for the writing mistakes. We have revised and marked it in blue in the revised manuscript. 
Comment 2: Introduction part: From line 15, there is a repetition of the word "the". From line 37, check the spelling of "mathacrvlate" and also add "an" before amorphous.

From line 55, replace described with describe. From lines 65 and 66, do you mean "time-domain"? From lines 78 and 79, please rephrase the sentence, what do you mean by "preformed"?

Response: We are very sorry for these writing mistakes. We have carefully checked the possible typos/spellings and grammar errors to improve quality of the writing in the whole paper. The improvements are marked in blue in the revised manuscript.

Comment 3: Add hyphen to the word "fractional order" in lines 18, 108, $113,116 \& 152$.

Response: We have added hyphen for all "fractional order" and marked it in blue in the revised manuscript.

Comment 4: Subscript "T" used in equations 26, 28, 43 and 48 should be defined.

Response: In mathematical expression, the superscript " $\mathrm{T}$ " represents the transpose of the matrix, there is no need to define it.

Comment 5: Check the word "discretize" in line 144.

Response: We have revised and marked in blue in the revised manuscript. 
The variable $(x, t)$ is discretized by the reasonable match points $x_{i}=\frac{2 i-1}{2(n+1)} H, i=1,2, \cdots n, t_{j}=\frac{2 j-1}{2(n+1)} S, j=1,2, \cdots n$, based on the collection method.

Comment 6: Can you buttress your eq. 50 with more explanation of how you obtained decimal number for partial derivative subscript (i.e. 4.1), subscript t (i.e. 0.1 and 1.9)?

Response: In order to prove the accuracy of the algorithm proposed in this paper, the numerical example is consistent with the governing equation form of the viscoelastic beam, and the parameters are different. The parameters of the numerical example are chosen to be arbitrary values. In order to compare the error between the algebraic solution and the numerical solution, we can derive the expression of $f(x, t)$ based on the algebraic solution of the equation, so as to find the numerical solution to compare with the algebraic solution.

Comment 7: Replace is with are in line 183.

Response: We have revised and marked in blue in the revised manuscript. Comment 8: From line 186, change "to" to "on" Response: We have revised and marked in blue in the revised manuscript. Comment 9: Check your Tables naming and your explanation of these tables, you must be consistent (i.e. if you are using table 1 for your table then use table 1, not Tab. 1 for your explanation. Also, if you are using 
Tab. 1 for your table then you can use Tab. 1 not Table 1 for your explanation and so on)

Response: We are very sorry for the difference between the table name and the explanation. We have revised the table name and explanation uniformly and marked it in blue in the revised manuscript.

Comment 10: Check your Figures naming and your explanation of these figures, consistency is very important (i.e. if you are using figure 1 for your diagram then use figure 1, not fig. 1 for your explanation. Also, if you are using fig. 1 for your table then you can use fig. 1 not figure 1 for your explanation and so on)

Response: We are very sorry for the difference between the Figures naming and explanation. We have revised the naming and explanation of the figure and marked it in blue in the revised manuscript.

Comment 11: Check the statement below eq. (47) line 3, should be written as "Legendre polynomials. Afterwards, the considered ..."

Response: We are very sorry for the error in segmentation. We have made revised and marked it in blue in the revised manuscript.

Comment 12: Check line 141, "... is determinate ..." This statement needs to be corrected.

Response: We have changed "is determinate" to "can be obtained".

Comment 13: Check lines $156 \&$ \& 165, "... determinated ..." This word is not in the dictionary rather "determined". 
Response: We are very sorry for these writing mistakes. We have carefully checked the possible typos/spellings and grammar errors to improve quality of the writing in the whole paper. We have changed "determinated" to "determined".

Comment 14: Below table 2, this statement " After to have ..." needs to be reconstructed.

Response: We have revised this sentence and marked it in blue in the revised manuscript.

Reviewer 2:

Comment 1: Please check the entire manuscript for grammatical errors/typos.

Response:We have carefully checked the possible typos/spellings and grammar errors to improve quality of the writing in the whole paper. Comment 2: In Eq. 1, $\alpha>0, m-1 \leq \alpha<m$ should be $\alpha>0, m-1<\alpha<m$. Response: We are very sorry for the error in Eq. 1. We have modified Eq. 1.

Comment 3: Just for verification, the authors can re-verify if the absolute value of $\mathrm{x}=1.6, \mathrm{t}=4$ and $\mathrm{n}=5$ is indeed $-0.0232 \times 10^{-9}$ in Table 1 .

Response: We are very sorry for this error, the absolute error value should be $0.0232 \times 10^{-9}$. 
Comment 4: The author needs to improve the introduction and talk also about analytical methods of solving fractional PDEs. For this purpose, the authors can add the following references to enrich the introductory section.

Response: We have revised the format of references and added relevant documents suggested by reviewers.

Reviewer 4:

Comment 1: The highlights are effective, however, the last highlight may be reworded.

Response: The last highlight has been revised to "Dynamic analysis of PMMA beam under different load conditions and temperatures".

Comment 2: In the first equation, the authors have defined one type of the Caputo fractional derivatives, however, there is another type. The authors either should add the full definition or should explain any type.

Response: In this paper, only the Caputo fractional derivatives are applied in the calculation. This is the reason why only the Caputo fractional operator is defined in Eq (1). It is not necessary to define and explain the other types of fractional derivative.

Comment 3: In the equation-5, equation- 6 and after that, the authors should explain what are the type of strain and stress. Are they tensors or 
less and if they are not tensors what about applying for the polymeric materials or other complex materials.

Response: The stress is uniaxial normal stress and the strain is normal strain. They are not tensors, because we are interested only the stress and strain in the uniaxial direction of the beam. PMMA used in this paper is isotropic viscoelastic material, which exhibits identical material properties in all directions at every given point.

Comment 4: The authors may use the complex elastic modulus instead of the complex modulus in all of the manuscript.

Response: We have revised all "complex modulus" to "complex elastic modulus" and marked them in cyan.

Comment 5: The proof of the equation-11 and the equation-12 must be illustrated.

Response: The proof process of the formula has been given and is marked in cyan in the revised manuscript.

Comment 6: In the most literatures, the symbol used for displacement is Y rather than $\omega$ and the authors are encouraged to use this.

Response: We have accepted the reviewer's suggestion to replace all $\omega(x, t)$ in the paper and figures with $y(x, t)$.

Comment 7: The authors used the same symbol $\mathrm{T}$ for two different things. In the equations from 19 and after that in Legendre polynomial and before that for kinetic energy. Also, the same thing is repeated with the 
symbols A which was used for three different aspects. The authors must reword the used symbols and use different symbols for different things. Response: In order to distinguish, We have used $E_{k}$ to represent kinetic energy and $T$ to represent temperature. We keep one thing of A, and the other two things of A are also represented by other symbols.

Comment 8: Before the algorithm of the numerical method, the authors state that they use Matlab software for fitting the coefficients with least square method. The authors should explain if they used the fitting functions found in Matlab software or if they created a program for this purpose.

Response: In order to obtain the coefficient matrix of the equation, we used the numerical algorithm in MATLAB platform. The fractional-order equation is transformed to algebraic equations. The least square method is used to calculate the solution of the algebraic equations. Thus the numerical solution of the fractional order governing equation is obtained. Comment 9: In the table-1, the authors should explain why they considered the used orders of the derivatives. Also, in the table-2, the authors should add more details about the experimental results of the elastic modulus of Polymethylmethacrylate and from any study.

Response: In this paper, $\mathrm{n}$ is the number of terms of the shifted Legendre polynomial. As $\mathrm{n}$ increases, the absolute error gradually decreases, indicating the accuracy of the algorithm. Some details about experimental 
results have been added and are marked in cyan in the revised manuscript.

Comment 10: The authors must add some recent studies about using the fractional derivatives for the polymeric materials and other complex materials in the materials science which are Physica A: Statistical Mechanics and its Applications Volume 545, 1 May2020,123763 where the authors studied the viscoelastic with hardening strain with fractional derivatives. Also, Heliyon Volume 6, Issue 7, July2020, e04495 where the authors studied polymeric materials with fractional derivatives without using Laplace transformation. The authors should add and discuss these studies. Also, Mechanics of Materials, 103506, 2020 where the authors studied the viscoelastic of foam with fractional derivatives.

Response: We have added references suggested by reviewers.

Comment 11: In the figure-2, the authors may used the traditional symbol for the frequency or if they mean this is the angular frequency, they have to change the unit.

Response: This paper used the traditional frequency and we have revised the symbol of the frequency.

Comment 12: In a lot of experimental works and engineering application the function $\mathrm{f}(\mathrm{x}, \mathrm{t})=\mathrm{A} \sin (100<\mathrm{pi}>\mathrm{t})$ instead of $\mathrm{f}(\mathrm{x}, \mathrm{t})=\mathrm{A} \sin (0.5 \mathrm{t})$ when the unit of the time is second. The authors should explain why they 
used this form. Also, in the same function, there is no need for the unit and the unit should be put after the values of $A$ in the same subsection. Response: According to the reviewer's comment, we modified the form of the simple harmonic load in the paper, and made corresponding changes to the relevant pictures.

Comment 13: The authors may add more details about expanding the method to other fluids and how it can be applied.

Response: In the conclusion, we added a paragraph to illustrate the significance and importance of the algorithm proposed in this paper.

Not only the unsuited contents have been revised one by one according to the reviewer's comments, but also the careful polishing has been done to the whole manuscript. Any changes haven been marked in the revised manuscript.

Thanks again for your help for our manuscript. 


\section{Summary of changes}

1. Page 1 , in the abstract line 12 , "determinated" has been revised to "determined".

2. Page 1, 4, 21, all "time domain" have been revised to "time-domain".

3. Page 2, 6, 13, 14, 22, all "fractional order" have been revised to “fractional-order".

4. Page 2, line 11, "Meng et al. [4] proposed a new variable-order fractional derivative viscoelastic model to describe the strain hardening behavior of amorphous glassy polymers, in which the order function was assumed to be linearly varied with time. The comparison between the model prediction and experimental data confirmed the close relationships of order change and strain hardening" has been added.

5. Page 2, "Henriques et al. [8] used the fractional derivative model to describe the viscoelastic behaviour of two polymeric foams. Excellent correlation between the experimental data and model predictions was observed for the shear storage modulus." has been added.

6. Page 2 , line 20 , delete a word "the".

7. Page 3 , line 45 , "mathacrvlate" has been revised to "methacrylate" and "an" has been added before "amorphous".

8. Page 3, line 63, "described" has been revised to "describe".

9. Page 4, "Akinyemi and Lyiola [18] used the Shehu transform method to decompose the nonlinear term to solve the time fractional differential 
equations in physics and engineering. Senol et al. [19] proposed an analytical approximate method based on residual power series and q-homotopy analysis method [20] to solve the nonlinear time fractional equations. Akinyemi et al. [21] developed a new iterative method with q-homotopy analysis to solve the fourth and sixth order time fractional Cahn-Hilliard equations. The simplicity and accuracy of the methods were confirmed by the provided examples. Al-Raeei and El-Daher [22] developed numerical-integral methods to find numerical solutions of the fractional Schrodinger equation without using Laplace transform." has been added.

10. Page 4, line 96, "In this paper, the dynamic analysis of the viscoelastic beam is preformed under various loading conditions" has been revised to "In this paper, the dynamic analysis of viscoelastic beam under various loading conditions is studied".

11. Page 4, line 99, "models are" has revised to "model is".

12. Page 5, " $\alpha>0, m-1 \leq \alpha<m$ " has revised to " $\alpha>0, m-1<\alpha<m$ ".

13. Page 6, Section 2.2, "constitution" has revised to "constitutive".

14. Page 7, 16, 17, 18, 20, all "Fig." have been revised to "Figure".

15. Page 7, "complex modulus" has revised to "complex elastic modulus".

16. Page 7, Eq. 9 has been rewritten.

17. Page 7, " $A$ is the cross-sectional area" has revised to " $A_{x}$ is the 
cross-sectional area".

18. Page 7, kinetic energy is redefined as $T^{\prime}$

19. Page 13, "The $\alpha$ exponent $t$ h derivative of $\phi_{n}(x)$ with respect to $t$ is formulated as" has revised to "The $\alpha^{\text {th }}$ derivative of $\phi_{n}(x)$ can be expressed as".

20. Page 13, "shifted Legendre polynomials, afterwards the considered" has revised to "shifted Legendre polynomials. Afterwards, the considered"

21. Page 14, line 174, "Based on the collection method, the reasonable match points $x_{i}=\frac{2 i-1}{2(n+1)} H, i=1,2, \cdots n, t_{j}=\frac{2 j-1}{2(n+1)} S, j=1,2, \cdots n$, have been used to discrete the variable $(x, t)$ to $\left(x_{i}, t_{j}\right)$." has revised to "The variable $(x, t)$ is discretized by the reasonable match points $x_{i}=\frac{2 i-1}{2(n+1)} H, i=1,2, \cdots n, t_{j}=\frac{2 j-1}{2(n+1)} S, j=1,2, \cdots n$, based on the collection method."

22. Page 14, line 177, "is determinate" has revised to "can be obtained".

23. Page 14, line 178, "using Matlab platform and least square method" has been revised to "using the numerical algorithm in MATLAB platform. The fractional-order equation is transformed to algebraic equations. The least square method is used to calculate the solution of the algebraic equations.".

24. Page 14, line 186, " in which the left term is similar to the governing 
equation of the viscoelastic beam (defined in Eq. (17)) and $f(x, t)$ is derived from algebraic solution." has been added.

25. Page 15, line 189, "determinated" has revised to "determined".

26. Page 15 , Table $1, "-0.0232 \times 10^{-9} "$ has revised to " $0.0232 \times 10^{-9} "$.

27. Page 15, line 191, "In this research, $\mathrm{n}$ is selected as 6 to reduce the absolute error in the calculation." has been added.

28. Page 15, line 199, "determinated" has revised to "determined"

29. Page 15, line 199, "using the dynamic mechanical analysis by" has been added.

30. Page 15, line 200, "The cylindrical compression samples were tested by using the harmonic solicitations with different frequency at various temperature." has been added.

31. Page 15, 17, 20, all "Tab." have been revised to "Table".

32. Page 15, "After to have implemented these identified parameters in Eq. (11) and (12), the values of the simulative modulus $E^{\prime}$ and $E^{\prime \prime}$ are in excellent agreements with the experimental modulus, see Figure 2" has deleted.

33. Page 16, Table. 2, "isotropic" has been added.

34. Page 17, line 216, "is" has been revised to "are".

35. Page 17, line 219, "to" has been revised to "on".

36. Page 17, before Eq. (53), "are" has been revised to "are as".

37. Page 17, line 225, "the time of uniform load loading" has revised to 
"the loading time of uniformly distributed load".

38. Page 18, line 231, “ $f(x, t)=A \sin (0.5 t) N / m$ " has revised to “ $f(x, t)=B \sin (100 \pi t) "$.

39. Page 18 , line $233, " A=100,200,500$ and $1000 "$ has revised to " $\mathrm{B}=$ $100,200,500$ and $1000 \mathrm{~N} / \mathrm{m} "$

40. Page 20, line 250, "As shown in these figure, the higher the temperature, the greater the displacement of the PMMA beam. These show the ability of the PMMA beam to resist loads decreases with increasing temperature and time." has revised to "Based on Figure. 6, the displacement of the beam increases with temperature. It indicates the ability of the PMMA beam to resist load decreases with increasing temperature and time."

41. Page 22, line 289, “The numerical algorithm proposed in this paper could be used to solve other engineering problems. The identification method of the viscoelastic properties is suitable for not only solid materials, but also fluid materials. Dynamic mechanical analysis is always used to characterize the viscoelastic properties of material. The parameters in the fractional viscoelastic behaviour law can be identified according to the experimental investigations. The governing equation of the problem, associated with the proper parameters of material behaviour, could be efficiently and accurately solved with the proposed numerical algorithm." has been added. 
42. Acknowledgements has been updated.

43. References have been updated.

44. Appendix have been updated. 


\section{Highlights:}

- An improved fractional viscoelastic constitutive model is proposed.

- The parameters in the proposed viscoelastic model are identified by DMA tests.

- An algorithm based on shifted Legendre polynomials is proposed with high accuracy.

- The fractional governing equation of the viscoelastic beam is solved directly in time domain.

- Dynamic analysis of PMMA beam under different load conditions and temperatures. 


\title{
Shifted Legendre polynomials algorithm used for the dynamic analysis of PMMA viscoelastic beam with an improved fractional model
}

\author{
Jiawei $\mathrm{Cao}^{\mathrm{a}}$, Yiming Chen ${ }^{\mathrm{a}, \mathrm{b}, * *}$, Yuanhui Wang ${ }^{\mathrm{a}, *}$, Gang Cheng ${ }^{\mathrm{c}}$, Thierry \\ Barrière $^{\mathrm{b}}$ \\ ${ }^{a}$ College of Sciences, Yanshan University, Qinhuangdao, 066004, Hebei, China \\ ${ }^{b}$ Univ. Bourgogne Franche-Comté, FEMTO-ST Institute, CNRS/ENSMM/UTBM, \\ Department of Applied Mechanics, 25000 Besançon, France \\ ${ }^{c}$ INSA Centre Val de Loire, Univ. Tours, Univ. Orléans, LaMé, 3 rue de la chocolaterie, \\ CS 23410, 41034 Blois, France
}

\begin{abstract}
In this paper, a fractional viscoelastic model is proposed to describe the physical behaviour of polymeric material. The material parameters in the model are characterized by the experimental data obtained in the dynamical mechanical analysis. The proposed model is integrated into the fractional governing equation of polymethyl methacrylate (PMMA) above its glass transition temperature. The numerical algorithm based on the shifted Legendre polynomials is retained to solve the fractional governing equations in the time-domain. The accuracy and effectiveness of the algorithm are verified according to the mathematical examples. The advantage of this method is that Laplace transform and the inverse Laplace transform commonly used in fractional calculus are avoided. The dynamical response of the viscoelastic PMMA beam is determined with several loading conditions (uniformly distributed load and harmonic load). The effects of the loading condition and the temperature on the dynamic response of the beam are investigated in the results. The proposed approach shows great potentials
\end{abstract}

\footnotetext{
${ }^{\star 2}$ Fully documented templates are available in the elsarticle package on CTAN.

* Corresponding author

** Corresponding author

Email addresses: chenym@ysu.edu.cn (Yiming Chen), wangyuanhui527@163.com (Yuanhui Wang)
} 
for the high-precision calculation in solving the fractional equations in the science and engineering.

Keywords: Fractional calculus, Fractional partial differential equation, Dynamic analysis, Polymethyl methacrylate, Viscoelastic model, Shifted Legendre polynomial

\section{Introduction}

Fractional calculus has been widely used in the fields of science and engineering in recent years. The fractional-order models of the materials are integrated into the integrals and differential equations to improve their 5 behaviour modelling efficiency and accuracy. Fractional calculus was used to investigate the viscoelastic behaviour of the fluid in the pipes [1] and the dynamic behaviour of the active elastomers in magnetic field [2]. Fractionalorder Constitutive Equation (FCE) was proposed to describe the physical behaviour of non-Newtonian fluid [3]. The results showed that the proposed equations could successfully capture the observed increasing of shear stress for different velocity gradients. Meng et al. [4] proposed a new variableorder fractional derivative viscoelastic model to describe the strain hardening behaviour of amorphous glassy polymers, in which the order function was assumed to be linearly varied with time. The comparison between the model prediction and experimental data confirmed the close relationships of order change and strain hardening. A variable-order Fractional Differential Equation (FDE) was developed to modelling the shape memory behaviour of the polymers [5], which proves more suitable than the conventional constant order FDE. A fractional model composed parallel fractional Maxwell elements was used to describe the mechanical behaviour of the polymers [6]. It was able to describe the evolution of the master curves of storage modulus and loss modulus during the stress relaxation experiments. A viscoelastic model employing fractional-order derivatives was applied to describe the dielectric properties of materials [7]. Henriques et al. [8] used the fractional derivative model to describe the viscoelastic behaviour of two polymeric foams. Excellent correlation between the experimental data and model predictions was observed for the shear storage modulus. Fractional calculus has shown great advantages in modelling the physical constitutive behaviours of materials, especially the viscoelastic properties of polymeric materials.

30 The viscoelastic beam is an important structural element to resist load in 
engineering. Many fractional material models have been proposed to analyze the dynamic response of the beam under various loading conditions. A fractional Kelvin-Voigt model was used to describe the viscoelastic properties of the beam. The numerical results revealed that the increase of the derivative order could cause a decrease in the vibration amplitudes of the beam [9]. The dynamic analysis of a simple supported viscoelastic beam with a fractional Zener model was performed by using a modified variational iteration method [10]. The numerical examples were effectuated to study the influences of fractional derivative on the dynamic response the structure. The comparison with the classical Zener model proved the efficiency of the proposed algorithm for solving the fractional governing equations. It is considered as an important issue to propose the efficient fractional constitutive viscoelastic models to describe accurately the physical constitutive behaviour of the beams in mechanical engineering.

45 Polymethyl methacrylate (PMMA) is an amorphous thermoplastic polymer, which is widely used in the manufacturing of medical devices due to its excellent mechanical and optical properties. The mechanical property of PMMA material above its glass transition temperature is greatly affected for further applications. Its mechanical properties above the glass transition temperature are greatly affected for further applications in the life prediction and design of engineering structures. Jo et al. [11] described the nonlinear tensile behaviours of PMMA foam by different integer viscoelastic models. The proposed constitutive equations were developed in terms of strain, strain rate, elastic modulus, relative density of foam and relaxation time constant. The nonlinear tensile stress-strain behaviours were well described. Varghese and Batra [12] utilized the modified viscoplastic constitutive equations to simulate the mechanical behaviour of PMMA at high strain rates. The proposed models were found to be well coherent with the experimental results available in the literature. Cheng et al. [13] used a generalized Maxwell glass transition temperature. The generalized Maxwell model parameters were determined via dynamic mechanical analysis. The proposed model was applied to describe the relaxation modulus to achieving the numerical simulation of the hot embossing process [14]. However, there is little research work concerning the fractional constitutive viscoelastic model for PMMA above its glass transition temperature.

A suitable numerical algorithm has to be proposed to solve the fractional governing equation of the beam established based on the viscoelastic material 
models. The ordinary numerical algorithms are developed by using the multi-scale method [15], Galerkin method [16], finite element method [17] and so on. One of the difficulties with these methods is that the timedomain solutions could not be obtained directly. The Laplace transform has to be used to transform time-domain fractional equations into frequency domain fractional equations. The time-domain solution is then obtained by applying the inverse Laplace transform to the frequency domain solutions. The complexity of Laplace and inverse transform makes it difficult to solve effectively the fractional equations. Akinyemi and Lyiola [18] used the Shehu transform method to decompose the nonlinear term to solve the time fractional differential equations in physics and engineering. Şenol et al. [19] proposed an analytical approximate method based on residual power series and q-homotopy analysis method [20] to solve the nonlinear time fractional equations. Akinyemi et al. [21] developed a new iterative method with q-homotopy analysis to solve the fourth and sixth order time fractional Cahn-Hilliard equations. The simplicity and accuracy of the methods were confirmed by the provided examples. Al-Raeei and El-Daher [22] developed numerical-integral methods to find numerical solutions of the fractional Schrödinger equation without using Laplace transform. This is the reason why the algorithms based on the polynomials are proposed to solve fractional differential equations. The polynomial algorithms can solve directly the fractional differential equations in the time-domain. They exhibit many other advantages such as high accuracy and low complexity [23]. The commonly used polynomial algorithms include the Legendre polynomial [24], the Bernstein polynomial [25,26] and the Chebyshev polynomial [27,28]. Therefore, the polynomial algorithm is proposed for solving the fractional governing equation for PMMA viscoelastic beams.

In this paper, the dynamic analysis of viscoelastic beam under various loading conditions is studied. An improved fractional viscoelastic model is proposed to describe the constitutive behaviour of PMMA above its glass transition temperature. The proposed model is characterized in different temperatures via dynamic mechanical analysis. The fractional governing equations of the beam are established by integrating the fractional viscoelastic model. A numerical algorithm based on the polynomial and wavelet method is proposed to solve directly in the time-domain. The shifted Legendre polynomials retained can resolve effectively and accurately the complex fractional differential equations over a larger interval. The displacement of the viscoelastic beam versus time and position are obtained. 
The fractional derivative, the fractional viscoelastic constitutive model and the governing equation of the viscoelastic beam are introduced in Section 2. The shifted Legendre polynomials are presented in Section 3. The numerical example is proposed to verify the accuracy of the algorithm in Section 4. The dynamic analysis of the PMMA beam is performed under various loading conditions and temperatures in Section 5. The research work is concluded in Section 6. A list of the symbols used in the paper is given in Appendix A.

\section{Preliminary knowledge and definitions}

\subsection{Caputo fractional derivative operator}

Definition 2.1 The Caputo fractional derivative operator $D_{x}^{\alpha}$ of order $\alpha$ is defined as [29,30]

$$
D_{x}^{\alpha} u(x)= \begin{cases}\frac{1}{\Gamma(m-\alpha)} \int_{0}^{x}(x-\tau)^{-\alpha+m-1} u^{(m)}(\tau) d \tau, & \alpha>0, m-1<\alpha<m \\ \frac{d^{(m)} u(x)}{d x^{m}}, & \alpha=m\end{cases}
$$

where $x \geq 0$, and $m \in N$ ( $N$ denotes positive integer $)$.

The gamma function, denoted by $\Gamma(\cdot)$ is defined as $\Gamma(z)=\int_{0}^{\infty} e^{-t} t^{z-1} d t$ for complex arguments with positive real part.

Several significant properties of the Caputo fractional derivative are shown as follows:

$$
D_{x}^{\alpha} C=0
$$

where $C$ is constant.

The Caputo fractional derivative is a linear operator.

$$
D_{x}^{\alpha}(\lambda(u(x)))=\lambda D_{x}^{\alpha} u(x)
$$

where $\lambda$ is constant.

The Caputo fractional derivative of the power function satisfies

$$
D_{x}^{\alpha} x^{m}= \begin{cases}0, & m=0 \\ \frac{\Gamma(m+1)}{\Gamma(m+1-\alpha)} x^{m-\alpha}, & m=1,2,3, \cdots\end{cases}
$$




\subsection{Fractional constitutive viscoelastic model}

The general form of conventional viscoelastic stress strain relationship is expanded in Taylor series as follows [31]:

$$
\sum_{r=0}^{n} p_{r} \frac{d^{r} \sigma(t)}{d t^{r}}=\sum_{r=0}^{n} q_{r} \frac{d^{r} \varepsilon(t)}{d t^{r}}
$$

where $\sigma(t)$ is stress, $\varepsilon(t)$ is strain, $r \in N$ ( $N$ denotes positive integer).

The traditional differential equations established by integer order operators are limited to describe the viscoelastic behaviour of the material in mechanical engineering. The fractional-order differential operators are widely used in fractional viscoelastic constitutive models thanks to their memory-dependent properties. Several fractional models such as Maxwell model [32], KelvinVoigt [33] and Zenner [34] models are applied to describe the viscoelastic properties. The current integer order viscoelastic model (Eq. (5)) could be transformed to fractional-order viscoelastic model by replacing the integer order derivatives $\frac{d^{r}}{d t^{r}}$ by the fractional derivative $\frac{d^{\alpha_{r}}}{d t^{\alpha_{r}}}$.

$$
\sum_{r=0}^{n} p_{\alpha_{r}} \frac{d^{\alpha_{r}} \sigma(t)}{d t^{\alpha_{r}}}=\sum_{r=0}^{n} q_{\beta_{r}} \frac{d^{\beta_{r}} \varepsilon(t)}{d t^{\beta_{r}}}
$$

where $\alpha_{r}, \beta_{r} \in R$ ( $R$ denotes real number).

For complex materials, choosing one or two items does not describe the properties of the material well. Therefore, one item is selected on the left side of Eq. (6), and three items are selected on the right side to represent the constitutive relationship of the viscoelastic material, which includes integer order and fractional-order derivatives. The more terms are taken, the closer the constitutive equation is to the actual situation. The following formula is selected as the constitutive equation in this paper.

$$
\sigma(t)=q_{\alpha} D_{t}^{\alpha} \varepsilon(t)+q_{0} \varepsilon(t)+q_{1} D_{t}^{2} \varepsilon(t)
$$

where $q_{\alpha}, q_{0}, q_{1} \in R, \alpha \in(0,1)$.

Taking Fourier transform of both sides of Eq. (7) yields

$$
\bar{\sigma}(w)=q_{\alpha}(i w)^{\alpha} \bar{\varepsilon}(w)+q_{0} \bar{\varepsilon}(w)+q_{1}(i w)^{2} \bar{\varepsilon}(w)
$$

where $w$ is frequency. 
The complex elastic modulus $E^{*}$ is expressed as:

$$
\begin{gathered}
E^{*}=\frac{\bar{\sigma}(w)}{\bar{\varepsilon}(w)}=q_{\alpha}(i w)^{\alpha}+q_{0}+q_{1}(i w)^{2} \\
=q_{\alpha} w^{\alpha} \cos \left(\frac{\pi}{2} \alpha\right)+i q_{\alpha} w^{\alpha} \sin \left(\frac{\pi}{2} \alpha\right)+q_{0}-q_{1} w^{2} \\
E^{*}=E^{\prime}+i E^{\prime \prime}
\end{gathered}
$$

where $E^{\prime}$ is storage modulus, corresponding the real part of $E^{*}, E^{\prime \prime}$ is loss modulus, corresponding the imaginary part of $E^{*}$.

So, $E^{\prime}$ and $E^{\prime \prime}$ are expressed as:

$$
\begin{gathered}
E^{\prime}=q_{\alpha} w^{\alpha} \cos \left(\frac{\pi}{2} \alpha\right)+q_{0}-q_{1} w^{2} \\
E^{\prime \prime}=q_{\alpha} w^{\alpha} \sin \left(\frac{\pi}{2} \alpha\right)
\end{gathered}
$$

\subsection{Fractional governing equations of viscoelastic beam}

The deformation diagram of the viscoelastic beam is shown in Figure 1 . The differential equation of motion of the viscoelastic beam is established according to Hamilton's principle [35]. The kinetic energy [36] of the beam is expressed as

$$
E_{k}=\frac{1}{2} \int_{0}^{H} \rho A_{x} \frac{\partial^{2} y(x, t)}{\partial t^{2}} d x
$$

where $\rho$ is the material density, $A_{x}$ is the cross-sectional area, $H$ is the length of the beam and $y(x, t)$ is the displacement.

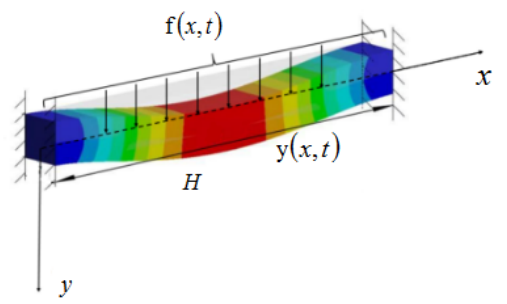

Figure 1: The geometric figure of the viscoelastic beam.

The potential energy [36] is expressed as

$$
V=\frac{1}{2} \int_{0}^{H}\left(q_{0} I \frac{\partial^{2} y(x, t)}{\partial x^{2}}+q_{\alpha} I \frac{\partial^{2+\alpha} y(x, t)}{\partial x^{2} \partial t^{\alpha}}+q_{1} I \frac{\partial^{4} y(x, t)}{\partial x^{2} \partial t^{2}}\right) d x
$$


where $I$ is the moment of inertia, $q_{\alpha}, q_{0}, q_{1}, \alpha$ are the identified parameters of the constitutive viscoelastic model.

The work done by the external forces is expressed as

$$
W=\frac{1}{2} \int_{0}^{H} f(x, t) y(x, t) d x
$$

where $f(x, t)$ is the external transverse axial load applied on the beam.

According to the Hamilton's principle

$$
\delta \int_{t_{1}}^{t_{2}}\left(E_{k}-V\right) d t+\int_{t_{1}}^{t_{2}} \delta W d t=0
$$

The fractional governing equation of motion of the viscoelastic beam is obtained:

$$
I\left(q_{\alpha} \frac{\partial^{4+\alpha} y(x, t)}{\partial x^{4} \partial t^{\alpha}}+q_{0} \frac{\partial^{4} y(x, t)}{\partial x^{4}}+q_{1} \frac{\partial^{6} y(x, t)}{\partial x^{4} t^{2}}\right)+\rho A_{x} \frac{\partial^{2} y(x, t)}{\partial t^{2}}=f(x, t)
$$

The beam is fixed at both ends and the boundary conditions are:

$$
y(x, 0)=0 \quad y(0, t)=0 \quad y(H, t)=0 \quad y_{x}(0, t)=0 \quad y_{x}(H, t)=0
$$

\section{Numerical algorithm of fractional governing equations}

\subsection{Shifted Legendre polynomials}

Legendre polynomials are orthogonal, which used as basic approximate functions. They exhibit simple and convenient form for calculation, compared with other orthogonal polynomials (Chebyshev polynomials...). The interval of Legendre polynomials is -1 to 1 . The shifted Legendre polynomials are defined to approximate the unknown function in a larger interval $[0, \mathrm{H}]$.

The shifted Legendre polynomial of degree $n$ in $[0,1]$ is defined as [37]

$$
l_{n, i}(x)=\sum_{k=0}^{n-i}(-1)^{k}\left(\begin{array}{c}
n-i \\
k
\end{array}\right)\left(\begin{array}{c}
n+i+k+1 \\
n-i
\end{array}\right) x^{i+k}
$$

where $i=0,1, \cdots, n, x \in[0,1]$. Then $\varphi_{n}(x)$ is formulated as

$$
\begin{gathered}
\varphi_{n}(x)=\left[l_{n, 0}(x), l_{n, 1}(x), \cdots, l_{n, n}(x)\right]^{T} \\
\varphi_{n}(x)=A T_{n}(x)
\end{gathered}
$$


where $T_{n}(x)=\left[1, x, \cdots, x^{n}\right]^{T}$,

$$
A=\left[a_{i j}\right]_{i, j=0}^{n}, \quad a_{i j}= \begin{cases}0, & 0 \leq k \leq i \\
(-1)^{k}\left(\begin{array}{c}
n-i \\
k
\end{array}\right)\left(\begin{array}{c}
n+i+k+1 \\
n-i
\end{array}\right), \quad k \leq i \leq n\end{cases}
$$

The shifted Legendre polynomial of degree $n$ in $[0, H]$ is formulated as

$$
\begin{aligned}
L_{n, i}(x) & =\sum_{k=0}^{n-i}(-1)^{k}\left(\begin{array}{c}
n-i \\
k
\end{array}\right)\left(\begin{array}{c}
n+i+k+1 \\
n-i
\end{array}\right)\left(\frac{x}{H}\right)^{i+k} \\
& =\sum_{k=0}^{n-i}(-1)^{k}\left(\begin{array}{c}
n-i \\
k
\end{array}\right)\left(\begin{array}{c}
n+i+k+1 \\
n-i
\end{array}\right)\left(\frac{1}{H}\right)^{i+k} x^{i+k},
\end{aligned}
$$

where $i=0,1, \cdots, n, x \in[0, H]$.

Then $\Phi_{n}(x)$ is formulated as

$$
\Phi_{n}(x)=A L T_{n}(x)
$$

where

$$
L=\left[l_{i j}\right]_{i, j=0}^{n}, \quad l_{i j}= \begin{cases}0, & i \neq j \\ H^{-i}, & i=j\end{cases}
$$

The shifted Legendre polynomial of degree $n$ in $[0, S]$ is formulated as

$$
\begin{aligned}
\bar{L}_{n, i}(t) & =\sum_{k=0}^{n-i}(-1)^{k}\left(\begin{array}{c}
n-i \\
k
\end{array}\right)\left(\begin{array}{c}
n+i+k+1 \\
n-i
\end{array}\right)\left(\frac{t}{S}\right)^{i+k} \\
& =\sum_{k=0}^{n-i}(-1)^{k}\left(\begin{array}{c}
n-i \\
k
\end{array}\right)\left(\begin{array}{c}
n+i+k+1 \\
n-i
\end{array}\right)\left(\frac{1}{S}\right)^{i+k} t^{i+k},
\end{aligned}
$$

where $i=0,1, \cdots, n, t \in[0, S]$.

Then $\phi_{n}(t)$ is defined as

$$
\phi_{n}(t)=A M T_{n}(t)
$$

where

$$
M=\left[m_{i j}\right]_{i, j=0}^{n}, \quad m_{i j}= \begin{cases}0, & i \neq j \\ S^{-i}, & i=j\end{cases}
$$




\subsection{Function approximation}

A continuous function $y(x)$ in the domain $[0, H]$ can be expanded in terms of shifted Legendre polynomials as $y(x)=\lim _{n \rightarrow \infty} \sum_{i=0}^{n} c_{i} L_{n, i}(x), y(x)$ can be approximated as

$$
y(x) \approx y_{n}(x)=\sum_{i=0}^{n} c_{i} L_{n, i}(x)=C^{T} \Phi_{n}(x)
$$

where $n$ is the number of terms of the shifted Legendre polynomial, $C^{T}=$ $\left[c_{0}, c_{1}, \cdots, c_{n}\right]$.

Then

$$
\begin{gathered}
C^{T}\left\langle\Phi_{n}(x), \Phi_{n}^{T}(x)\right\rangle=\left\langle y(x), \Phi_{n}^{T}(x)\right\rangle \\
C^{T}=\left\langle y(x), \Phi_{n}^{T}(x)\right\rangle Q^{-1} \\
Q=\left\langle\Phi_{n}(x), \Phi_{n}^{T}(x)\right\rangle=\left[\delta_{i j}\right]_{i, j=0}^{n}
\end{gathered}
$$

where $\delta_{i j}=\int_{0}^{H} L_{n, i}(x) L_{n, j}(x) d x=\left\{\begin{array}{ll}0, & i \neq j \\ \frac{H}{i+j+1}, & i=j\end{array}(i, j=0,1, \cdots, n)\right.$.

Similarly, a continuous function $y(t)$ in the domain $[0, S]$ can be expanded in terms of shifted Legendre polynomials as $y(t)=\lim _{n \rightarrow \infty} \sum_{i=0}^{n} k_{i} \bar{L}_{n, i}(t), y(t)$ can be approximated as

$$
y(t) \approx y_{n}(t)=\sum_{i=0}^{n} k_{i} \bar{L}_{n, i}(t)=K^{T} \phi_{n}(t)
$$

where $K^{T}=\left[k_{0}, k_{1}, \cdots, k_{n}\right]$.

Then

$$
\begin{gathered}
K^{T}\left\langle\Phi_{n}(t), \Phi_{n}^{T}(t)\right\rangle=\left\langle y(t), \Phi_{n}^{T}(t)\right\rangle \\
K^{T}=\left\langle y(t), \Phi_{n}^{T}(t)\right\rangle P^{-1} \\
P=\left\langle\Phi_{n}(t), \Phi_{n}^{T}(t)\right\rangle=\left[\Delta_{i j}\right]_{i, j=0}^{n}
\end{gathered}
$$

where $\Delta_{i j}=\int_{0}^{S} \bar{L}_{n, i}(t) \bar{L}_{n, j}(t) d t=\left\{\begin{array}{ll}0, & i \neq j \\ \frac{S}{i+j+1}, & i=j\end{array}(i, j=0,1, \cdots, n)\right.$. 
Two-variable continuous function $y(x, t) \in L^{2}[0, H] \times[0, S]$ can be formulated as

$$
\begin{aligned}
y(x, t) & =\lim _{n \rightarrow \infty} \sum_{j=0}^{n}\left(\sum_{i=0}^{n} c_{i} L_{n, i}(x)\right) k_{j} \bar{L}_{n, j}(t) \\
& =\lim _{n \rightarrow \infty} \sum_{j=0}^{n} \sum_{i=0}^{n} c_{i} k_{j} L_{n, i}(x) \bar{L}_{n, j}(t) \\
& =\lim _{n \rightarrow \infty} \sum_{j=0}^{n} \sum_{i=0}^{n} \omega_{i j} L_{n, i}(x) \bar{L}_{n, j}(t) \\
& =\lim _{n \rightarrow \infty}\left(\Phi_{n}(x) U \phi_{n}(t)\right)
\end{aligned}
$$

After intercepting the finite term, it can get

$$
y(x, t) \approx \Phi_{n}(x) U \phi_{n}(t)
$$

165 where $U=\left[y_{i j}\right]_{i, j=0}^{n}$ is a matrix of displacement functions.

\subsection{Integer order operator matrix}

The derivative of $\Phi_{n}(x)$ with respect to $x$ is formulated as

$$
\frac{d \Phi_{n}(x)}{d x}=D_{x} \Phi_{n}(x)
$$

Then

$$
D_{x} \Phi_{n}(x)=D_{x} A L T_{n}(x)=A L \frac{d T_{n}(x)}{d x}=A L E T_{n}(x)=A L E(A L)^{-1} \Phi_{n}(x)
$$

where $E=\left[e_{i j}\right]_{i, j=0}^{n}, \quad e_{i j}= \begin{cases}0, & i \neq j+1 \\ i, & i=j+1\end{cases}$

From Eq. (32), $D_{x}$ is obtained as

$$
D_{x}=A L E(A L)^{-1}
$$

The $m$ exponent th derivative of $\Phi_{n}(x)$ with respect to $x$ is formulated as

$$
\frac{d^{m} \Phi_{n}(x)}{d x^{m}}=D_{m x} \Phi_{n}(x), \quad m \in N
$$


Then

$D_{m x} \Phi_{n}(x)=D_{m x} A L T_{n}(x)=A L \frac{d^{m} T_{n}(x)}{d x^{m}}=A L E^{m} T_{n}(x)=A L E^{m}(A L)^{-1} \Phi_{n}(x)$

Based on Eq. (35), $D_{m x}$ can be obtained as

$$
D_{m x}=A L E^{m}(A L)^{-1}
$$

Similarly, $\phi_{n}(t)$ is a series of polynomial matrices with respect to $t$, the derivative of $\phi_{n}(t)$ with respect to $t$ is formulated as

$$
\frac{d \phi_{n}(t)}{d t}=D_{t} \phi_{n}(t)
$$

Then

$$
D_{t} \phi_{n}(t)=D_{t} A M T_{n}(t)=A M \frac{d T_{n}(t)}{d t}=A M E T_{n}(t)=A M E(A M)^{-1} \phi_{n}(t)
$$

Therefore, from Eq. (38), $D_{t}$ is obtained as

$$
D_{t}=A M E(A M)^{-1}
$$

The $v$ exponent th derivative of $\phi_{n}(t)$ with respect to $t$ is formulated as

$$
\frac{d^{v} \phi_{n}(t)}{d t^{v}}=D_{v t} \phi_{n}(t), \quad v \in N
$$

Then

$$
D_{v t} \phi_{n}(t)=D_{v t} A M T_{n}(t)=A M \frac{d^{v} T_{n}(t)}{d t^{v}}=A M E^{v} T_{n}(t)=A M E^{v}(A M)^{-1} \phi_{n}(t)
$$

From Eq. 41, $D_{v t}$ is obtained as

$$
D_{v t}=A M E^{v}(A M)^{-1}
$$

Thus,

$$
\frac{\partial^{m+v} y(x, t)}{\partial x^{m} \partial t^{v}} \approx\left(\left(D_{m x} \Phi_{n}(x)\right)^{T} U\left(D_{v t} \phi_{n}(t)\right)\right)
$$

where only $U$ is unknown. 
170

\subsection{Fractional-order operator matrix}

The $\alpha^{t h}$ derivative of $\phi_{n}(t)$ can be expressed as:

$$
\begin{gathered}
\frac{d^{\alpha} \phi_{n}(t)}{d t^{\alpha}}=D_{\alpha t} \phi_{n}(t), \quad \alpha \in(0,1) \\
D_{\alpha t} \phi_{n}(t)=D_{\alpha t} A M T_{n}(t)=A M \frac{d^{\alpha} T_{n}(t)}{d t^{\alpha}}=A M F T_{n}(t)=A M F(A M)^{-1} \phi_{n}(t) \\
\text { where } F=\left[f_{i j}\right]_{i, j=0}^{n}, \quad f_{i j}= \begin{cases}\frac{\Gamma(i+1)}{\Gamma(i+1-\alpha)} t^{-\alpha}, & i=j, i \geq 1 \\
0, & \text { otherwise }\end{cases}
\end{gathered}
$$

In view of Eq. (45), it gives that

$$
D_{\alpha t}=A M F(A M)^{-1}
$$

Thus,

$$
\frac{\partial^{m+\alpha} y(x, t)}{\partial x^{m} \partial t^{\alpha}} \approx\left(\left(D_{m x} \Phi_{n}(x)\right)^{T} U\left(D_{\alpha t} \phi_{n}(t)\right)\right)
$$

where only $U$ is unknown.

Basic of the above work, the unknown function is replaced with the approximate function containing differential operator matrix of shifted Legendre polynomials. Afterwards, the considered fractional governing Eq. (17) will be converted into the following algebraic equation with unknown $U$.

$$
\begin{aligned}
& I\left(q_{\alpha}\left(\left(D_{4 x} \Phi_{n}(x)\right)^{T} U\left(D_{\alpha t} \phi_{n}(t)\right)\right)+q_{0}\left(\left(D_{4 x} \Phi_{n}(x)\right)^{T} U\left(\phi_{n}(t)\right)\right)\right. \\
& +q_{1}\left(\left(D_{4 x} \Phi_{n}(x)\right)^{T} U\left(D_{2 t} \phi_{n}(t)\right)\right)+\rho A_{x}\left(\left(\Phi_{n}(x)\right)^{T} U\left(D_{\alpha t} \phi_{n}(t)\right)\right)=f(x, t)
\end{aligned}
$$

The boundary conditions Eq. (18) can be transformed into the following form:

$$
\begin{aligned}
& \Phi_{n}(x) U \phi_{n}(0)=0, \Phi_{n}(0) U \phi_{n}(t)=0, \Phi_{n}(H) U \phi_{n}(t)=0 \\
& D_{x} \Phi_{n}(0) U \phi_{n}(t)=0, D_{x} \Phi_{n}(H) U \phi_{n}(t)=0
\end{aligned}
$$

The variable $(x, t)$ is discretized by the reasonable match points $x_{i}=$ $175 \frac{2 i-1}{2(n+1)} H, i=1,2, \cdots, n, t_{j}=\frac{2 j-1}{2(n+1)} S, j=1,2, \cdots, n$, based on the collection method. Eq. (48) is transformed into a set of algebraic equations. 
The coefficient $y_{i j}(i=0,1,2, \cdots n ; j=0,1,2, \cdots n)$ can be obtained by using the numerical algorithm in MATLAB platform. The fractional-order equation is transformed to algebraic equations. The least square method is used to calculate the solution of the algebraic equations. The numerical solution of the fractional derivative equation can be obtained.

The proposed algorithm can be summarized as follows:

\begin{tabular}{lc}
\hline \multicolumn{2}{c}{ Algorithm: Numerical solution for the fractional governing equation } \\
\hline $\begin{array}{l}\text { Input: } \\
\text { Output: }\end{array}$ & $\alpha, q_{\alpha}, q_{0}, q_{1}, \rho, A_{x}, I, f(x, t)$ \\
1. & Function approximation $y(x, t) \approx \Phi_{n}(x) U \phi_{n}(t)$ \\
2. & Derive calculation of integer and fractional operator matrices \\
3. & Substitute the operator matrix into the initial equation \\
4. & The initial equation is transformed into an algebraic equation \\
5. & Let $x_{i}=\frac{2 i-1}{2(n+1)} H, i=1,2, \cdots, n, t_{j}=\frac{2 j-1}{2(n+1)} S, j=1,2, \cdots, n$ \\
6. & Solve algebraic equations with MATLAB mathematical software \\
7. & Obtain the solution of the initial equation $y(x, t)$ \\
\hline
\end{tabular}

\section{Numerical example}

In this section, numerical example is given to demonstrate the effectiveness and accuracy of the algorithm in solving fractional-order equations.

The following equation is considered as a numerical example, in which the left term is similar to the governing equation of the viscoelastic beam (defined in Eq. (17)) and $f(x, t)$ is derived from algebraic solution.

$$
\begin{gathered}
2 x^{3}\left(\frac{\partial^{4.1} y(x, t)}{\partial x^{4} \partial t^{0.1}}+\frac{\partial^{4} y(x, t)}{\partial x^{4}}+\frac{\partial^{6} y(x, t)}{\partial x^{4} \partial t^{2}}\right)+x^{2} \frac{\partial^{2} y(x, t)}{\partial t^{2}}=f(x, t) \\
f(x, t)=48 \frac{\Gamma(3)}{\Gamma(2.9)} x^{3} t^{1.9}+48 x^{3} t^{2}+96 x^{3}+2 x^{4}(2-x)^{2}
\end{gathered}
$$

where the defined boundary conditions as follows:

$$
y(x, 0)=0 \quad y(0, t)=0 \quad y(2, t)=0 \quad y_{x}(0, t)=0 \quad y_{x}(2, t)=0
$$

The algebraic solution of Eq. (50) is giving by the following equation:

$$
y(x, t)=x^{2}(2-x)^{2} t^{2}, \quad x \in[0,2], t \in[0,10]
$$


The numerical solutions of Eq. (50) are obtained by the proposed method. The solutions are calculated with different $n$ to validate the efficiency of the method. The values of absolute error between the algebraic and approximate solutions are determined at different $x$ and $t$, which is summarized in Table 1. The absolute errors are approximately $10^{-8}$. The absolute error decrease with $n$. In this research, $n$ is selected as 6 to reduce the absolute error in the calculation.

Eq. (50) is in the same fractional form of the fractional governing equation of viscoelastic beam. The right term of Eq. (50) is more general and complex than uniformly distributed load.

Table 1: Evolution of absolute error in function of $n$.

\begin{tabular}{ccccc}
\hline \multirow{2}{*}{$(x, t)$} & \multirow{2}{*}{ Algebraic solution } & \multicolumn{3}{c}{ Absolute error value } \\
\cline { 3 - 5 } & & $n=4$ & $n=5$ & $n=6$ \\
\hline$(0,0)$ & 0 & $0.0140 \times 10^{-8}$ & $0.0913 \times 10^{-9}$ & $0.0236 \times 10^{-9}$ \\
$(0,2)$ & 0 & $0.0221 \times 10^{-8}$ & $0.1765 \times 10^{-9}$ & $0.0941 \times 10^{-9}$ \\
$(0,4)$ & 0 & $0.0561 \times 10^{-8}$ & $0.0938 \times 10^{-9}$ & $0.0508 \times 10^{-9}$ \\
$(0,6)$ & 0 & $0.0488 \times 10^{-8}$ & $0.2287 \times 10^{-9}$ & $0.0765 \times 10^{-9}$ \\
$(0,8)$ & 0 & $0.0956 \times 10^{-8}$ & $0.5249 \times 10^{-9}$ & $0.0980 \times 10^{-9}$ \\
$(1.6,0)$ & 0 & $0.0109 \times 10^{-8}$ & $0.0268 \times 10^{-9}$ & $0.0208 \times 10^{-9}$ \\
$(1.6,2)$ & 1.6384 & $0.0066 \times 10^{-8}$ & $0.0615 \times 10^{-9}$ & $0.0100 \times 10^{-9}$ \\
$(1.6,4)$ & 6.5536 & $0.0474 \times 10^{-8}$ & $0.0232 \times 10^{-9}$ & $0.0158 \times 10^{-9}$ \\
$(1.6,6)$ & 14.7456 & $0.0221 \times 10^{-8}$ & $0.1650 \times 10^{-9}$ & $0.0528 \times 10^{-9}$ \\
$(1.6,8)$ & 26.2144 & $0.0780 \times 10^{-8}$ & $0.2350 \times 10^{-9}$ & $0.0096 \times 10^{-9}$ \\
\hline
\end{tabular}

\section{Results and discussions}

\subsection{Determination of material parameters}

The values of $E^{\prime}$ and $E^{\prime \prime}$ at different temperatures and frequency were determined by using the dynamic mechanical analysis by Cheng et al. [14]. The cylindrical compression samples were tested by using the harmonic solicitations with different frequency at various temperatures. The parameters in the viscoelastic constitutive equation are identified based on $E^{\prime}$ and $E^{\prime \prime}$ at $T=110^{\circ} \mathrm{C}, 115^{\circ} \mathrm{C}, 120^{\circ} \mathrm{C}$ and $125^{\circ} \mathrm{C}$.

The identified parameters $q_{\alpha}, q_{0}, q_{1}, \alpha$ were obtained by inverse method

with a least square regression according to Eq. (11) and (12). The identified parameters are summarized in Table 2 . 
Table 2: Identified parameters of the isotropic viscoelastic constitutive behaviour law at different temperatures: $T=110,115,120,125^{\circ} \mathrm{C}$

\begin{tabular}{ccccc}
\hline$T /{ }^{\circ} \mathrm{C}$ & $q_{\alpha} / \mathrm{MPa}$ & $q_{0} / \mathrm{MPa}$ & $q_{1} / \mathrm{MPa}$ & $\alpha$ \\
\hline 110 & 275.8275 & 582.8400 & -0.0002 & 0.1674 \\
115 & 760.7903 & -139.9032 & 0.0001 & 0.0966 \\
120 & 2181.6523 & -1939.0033 & 0.0000 & 0.0509 \\
125 & 1187.7101 & -1123.1952 & -0.0001 & 0.0836 \\
\hline
\end{tabular}

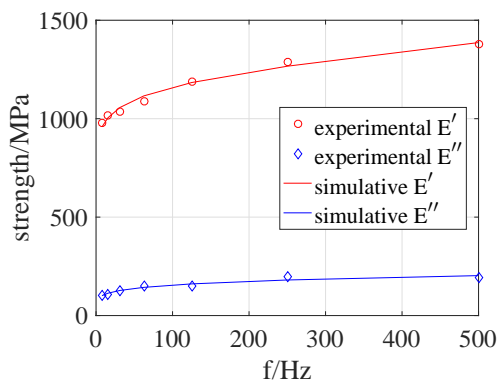

(a) $\mathrm{T}=110^{\circ} \mathrm{C}$

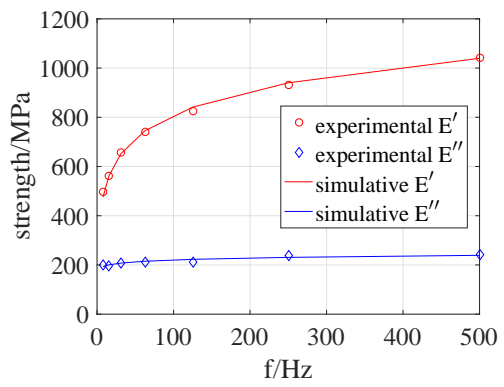

(c) $\mathrm{T}=120^{\circ} \mathrm{C}$

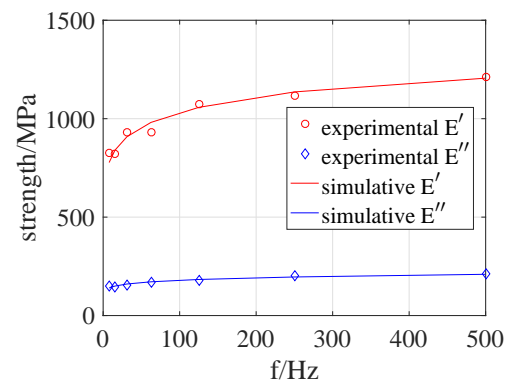

(b) $\mathrm{T}=115^{\circ} \mathrm{C}$

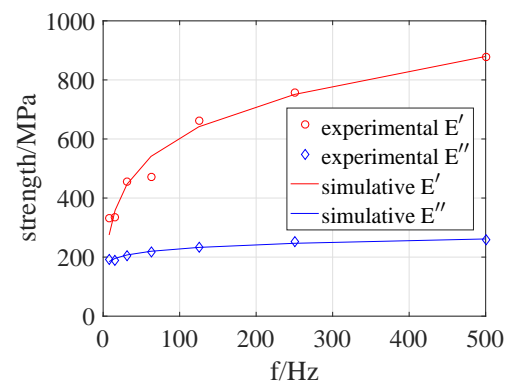

(d) $\mathrm{T}=125^{\circ} \mathrm{C}$

Figure 2: Comparison of identified viscoelastic behaviour of PMMA and the experimental date at different temperatures: (a) $\mathrm{T}=110^{\circ} \mathrm{C}$, (b) $\mathrm{T}=115^{\circ} \mathrm{C}$, (c) $\mathrm{T}=120^{\circ} \mathrm{C}$, (d) $\mathrm{T}=125^{\circ} \mathrm{C}$

Based on Figure 2, a good coherence between the experimental data and simulation data is observed in different temperatures. It indicates that the proposed fractional viscoelastic can describe accurately the mechanical 

parameters are defined in Table 2 .

Table 3: Input geometric and material parameters of the beam.

\begin{tabular}{cccccc}
\hline$H(\mathrm{~m})$ & $\rho\left(\mathrm{kg} / \mathrm{m}^{3}\right)$ & $A_{x}\left(\mathrm{~m}^{2}\right)$ & $I\left(\mathrm{~m}^{4}\right)$ & $T\left({ }^{\circ} \mathrm{C}\right)$ & $t(\mathrm{~s})$ \\
\hline 1.6 & $1.15 \times 10^{3}$ & 0.02 & $4.16 \times 10^{-6}$ & 110 & 60 \\
\hline
\end{tabular}

Based on the geometric and material parameters of the beam, Eq. (17) is developed as follows:

$$
\begin{gathered}
\frac{25}{6}\left(q_{\alpha} \frac{\partial^{4+\alpha} y(x, t)}{\partial x^{4} \partial t^{\alpha}}+q_{0} \frac{\partial^{4} y(x, t)}{\partial x^{4}}+q_{1} \frac{\partial^{6} y(x, t)}{\partial x^{4} \partial t^{2}}\right) \\
+23 \frac{\partial^{2} y(x, t)}{\partial t^{2}}=f(x, t)
\end{gathered}
$$

The boundary and initial conditions are as follows:

$$
\begin{gathered}
y(x, 0)=0 \quad y(0, t)=0 \quad y(1.6, t)=0 \quad y_{x}(0, t)=0 \\
y_{x}(1.6, t)=0 \quad, \quad x \in[0,1.6], t \in[0,60]
\end{gathered}
$$

\subsubsection{Uniformly distributed load}

The uniform transverse distributed load $100 \mathrm{~N} / \mathrm{m}$ is imposed on the beam. The numerical solution of the displacement of the beam is obtained by using the proposed algorithm.

The displacement of the beam at different $t$ and $x$ are shown in Figure 3 . The displacement curves at $t=6,12$ and $60 \mathrm{~s}$, exhibit the maximum value in the middle of the beam and zero value at both ends of the beam.

Further analysis shows that the loading time of uniformly distributed load has an effect on the dynamic displacement of the PMMA beam. As time 
increases, the dynamic displacement of the PMMA beam gradually increases. In engineering, this work provides a theoretical basis for the protection of load-bearing structures.

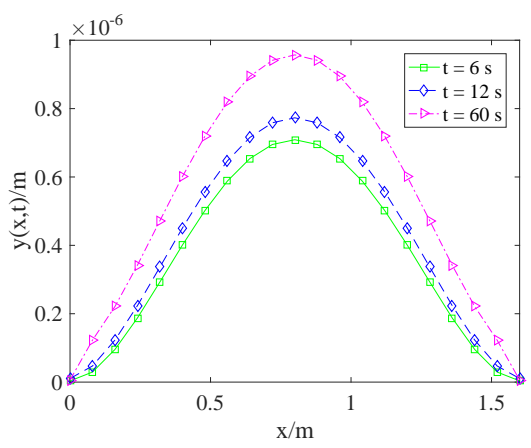

Figure 3: The dynamic displacement at different displacements and times.

\subsubsection{Harmonic load}

The harmonic load $f(x, t)=B \sin (100 \pi t)$ is applied on the viscoelastic beam. The transverse displacement of the viscoelastic beam is obtained with $B=100,200,500$, and $1000 \mathrm{~N} / \mathrm{m}$, as shown in Figure 4(a), Figure 4(b) and Figure 4(c), Figure 4(d) respectively.

235 The displacement of the two ends of a PMMA beam is always zero and is not affected by time, which is coherent with the boundary conditions. The maximum values of the displacement increase with the amplitude of the harmonic load. The curves of displacement of the beam under harmonic load with different amplitude are shown in Figure 4.

The displacements of the beam are calculated at the same position with different $t$ as shown in Figure 5. The maximal displacement of the beam increases with the amplitude of harmonic load. The larger amplitude of the harmonic load results in larger dissipation of the displacement. 


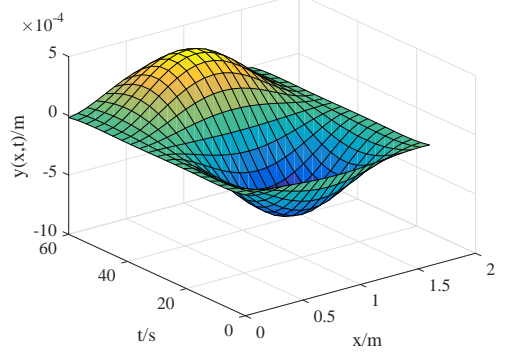

(a) $B=100 \mathrm{~N} / \mathrm{m}$

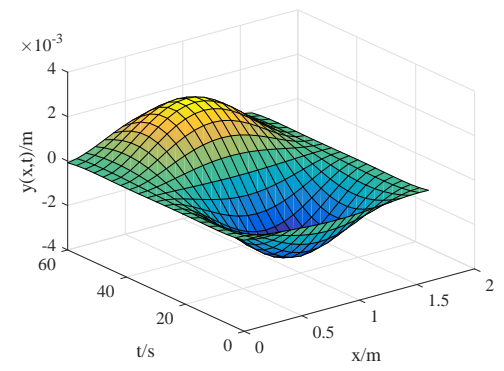

(c) $B=500 \mathrm{~N} / \mathrm{m}$

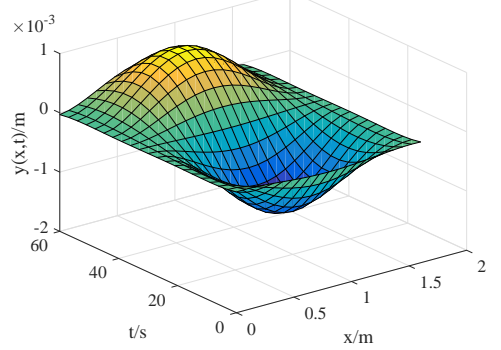

(b) $B=200 \mathrm{~N} / \mathrm{m}$

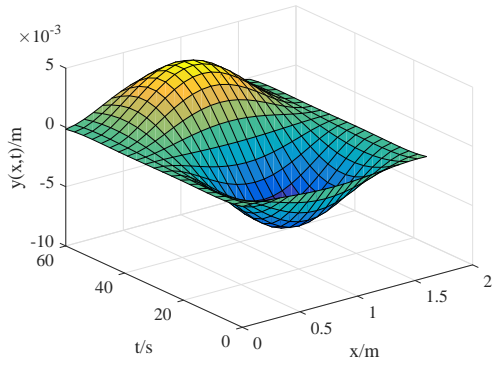

(d) $B=1000 \mathrm{~N} / \mathrm{m}$

Figure 4: The displacement of PMMA beam under different harmonic loads.

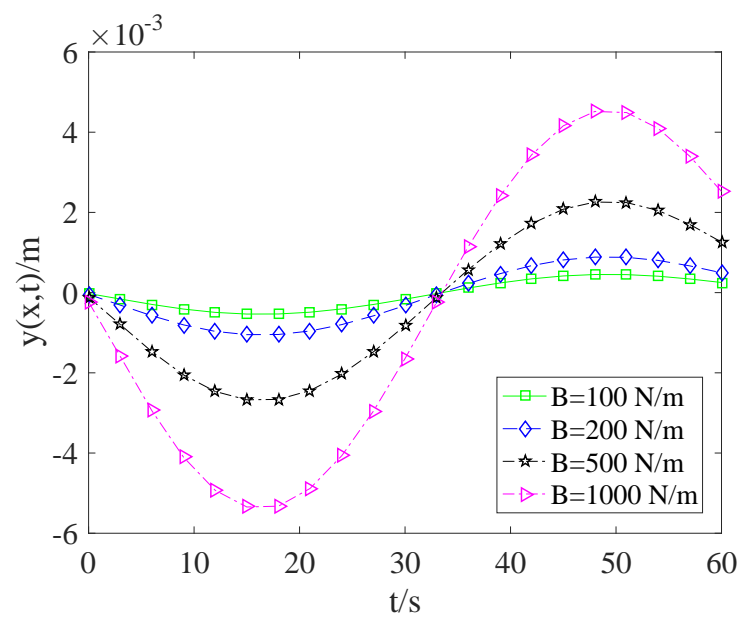

Figure 5: The displacement of PMMA beam under different harmonic loads when $x=0.8 \mathrm{~m}$. 


\subsection{Temperature effects on the dynamic response of viscoelastic beam}

The constitutive viscoelastic behaviour law were identified by the experimental data at different temperatures, as shown in Table 2.

Under $f(x, t)=100 \sin (100 \pi t)$, the numerical solutions $y(x, t)$ at different points for $T=110^{\circ} \mathrm{C}, 115^{\circ} \mathrm{C}, 120^{\circ} \mathrm{C}$ and $125^{\circ} \mathrm{C}$ are shown in Figure $6(\mathrm{a})$, Figure 6(b), Figure 6(c), and Figure 6(d) respectively.

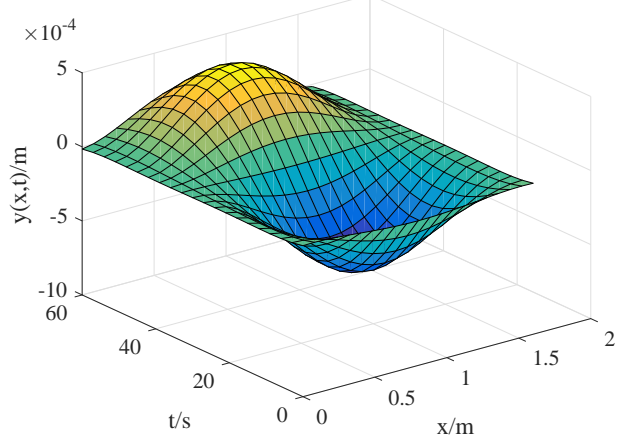

(a) $\mathrm{T}=110^{\circ} \mathrm{C}$

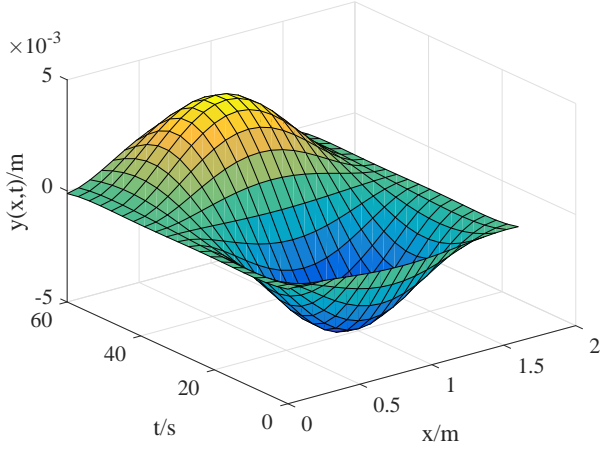

(c) $\mathrm{T}=120^{\circ} \mathrm{C}$

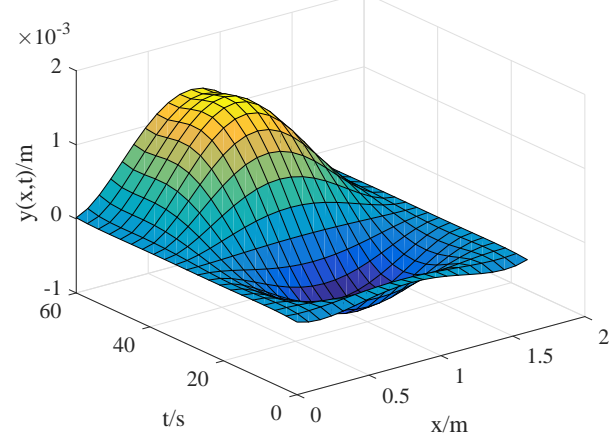

(b) $\mathrm{T}=115^{\circ} \mathrm{C}$

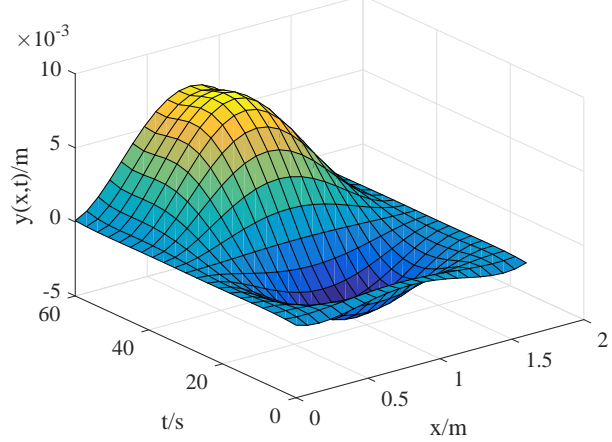

(d) $T=125^{\circ} \mathrm{C}$

Figure 6: The dynamic displacement $y(x, t)$ at different points under $100 \sin \left(100 \pi t\right.$ ) for (a) $\mathrm{T}=110^{\circ} \mathrm{C}$, (b) $\mathrm{T}=115^{\circ} \mathrm{C}$, (c) $\mathrm{T}=120^{\circ} \mathrm{C}$, and (d) $\mathrm{T}=125^{\circ} \mathrm{C}$.

Based on Figure6, the displacement of the beam increases with temperature. It indicates the ability of the PMMA beam to resist load decreases with increasing temperature and time. 
These results show that the temperature rising leads to the internal structure changes of the material, also the PMMA beam is weakened against the external deformation and the dynamic response becomes more obvious with time varying as the temperature rising.

\section{Conclusions}

In this paper, the shifted Legendre polynomials is used to solve the governing equation of PMMA viscoelastic beam directly in the time-domain. An improved fractional model is proposed to simulate the constitutive relationship of PMMA above the glass transition temperature. The fractional derivative model is used to describe the viscoelastic behaviour of the beam to predict the behaviour of the beam under uniform and simple harmonic loads. The numerical solution of PMMA viscoelastic beam displacement is obtained. At the same time, the displacements of PMMA beams at different temperatures are analyzed.

1. It is verified that the presented algorithm is accurate and effective through numerical example, and is also found that errors are decreasing with increasing $n$. Further, the shifted Legendre polynomials algorithm is excepted to solve fractional differential equations with three and more variables. More importantly, the proposed algorithm has shown great potentials for highprecision engineering computing problems.

2. The improved fractional-order model is used to derive the fractionalorder governing differential equations of viscoelastic beams, and the dynamic characteristics of viscoelastic materials are studied. It fills the blank in the field of high precision constitutive model simulation of PMMA materials at glass transition temperature.

3. Analysis shows that the time of uniform load affects the dynamic displacement of PMMA beams. With the time increasing, the dynamic displacement is increasing cumulatively in a fluctuating manner. In engineering, this work provides a theoretical basis for the protection of load-bearing structures above glass transition temperature.

4. The temperature rising leads to the internal structure changes of the material, also the PMMA beam is weakened against with the external deformation and the dynamic response becomes more obvious with time varying as the temperature rising. The results show that as the temperature increases and the time prolongs, the load resistance of PMMA beam gradually decreases. 
The numerical algorithm proposed in this paper could be used to solve

other engineering problems. The identification method of the viscoelastic properties is suitable for not only solid materials, but also fluid materials. Dynamic mechanical analysis is always used to characterize the viscoelastic properties of material. The parameters in the fractional viscoelastic behaviour law can be identified according to the experimental investigations. The governing equation of the problem, associated with the proper parameters of material behaviour, could be efficiently and accurately solved with the proposed numerical algorithm.

\section{Acknowledgements}

This work is supported by the Natural Science Foundation of Hebei Province (A2017203100) in China and the LE STUDIUM RESEARCH PROFESSORSHIP award of Centre-Val de Loire region in France. This work has also been supported by the EIPHI Graduate School (contract ANR-17EURE-0002).

\section{References}

[1] Wang XP, Qi HT, Yu B, Xiong Z, Xu HY. Analytical and numerical study of electroosmotic slip flows of fractional second grade fluids. Communications Nonlinear Science and Numerical Simulation, 2017;50:7787.

[2] Nadzharyan TA, Kostrov SA, Stepanov GV, Kramarenko EY. Fractional rheological models of dynamic mechanical behavior of magnetoactive elastomers in magnetic fields. Polymer, 2018;142:316-329.

[3] Sun HG, Zhang Y, Wei S, Zhu JT, Chen W. A space fractional constitutive equation model for non-Newtonian fluid flow. Communications Nonlinear Science and Numerical Simulation, 2018;62:409-417.

[4] Meng RF, Yin DS, Yang HX, Xiang GJ. Parameter study of variable order fractional model for the strain hardening behavior of glassy polymers. Physica A: Statal Mechanics and its Applications, 2020;545:123763.

[5] Li Z, Wang H, Xiao R, Yang S. A variable-order fractional differential equation model of shape memory polymers. Chaos, Solitons \& Fractals, 2017;102:473-485. 
[6] Lei D, Liang YJ, Xiao R. A fractional model with parallel fractional Maxwell elements for amorphous thermoplastics. Physica A, 2018;490:465475.

[7] Wharmby AW, Bagley RL. Modifying Maxwell's equations for

325

330

dielectric materials based on techniques from viscoelasticity and concepts from fractional calculus. International Journal of Engineering Science, 2014;79:59-80.

[8] Henriques IR, Rouleau L, Castello DA, Borges LA, Deü JF. Viscoelastic behavior of polymeric foams: Experiments and modeling. Mechanics of Materials, 2020;148:103506.

[9] Freundlich J. Transient vibrations of a fractional Kelvin-Voigt viscoelastic cantilever beam with a tip mass and subjected to a base excitation. Journal of Sound and Vibration, 2019;438:99-115.

[10] Martin O. Stability approach to the fractional variational iteration method used for the dynamic analysis of viscoelastic beams. Journal of Computational and Applied Mathematics, 2019;346:261-276.

[11] Jo C, Fu J, Naguib HE. Constitutive modeling for mechanical behaviour of PMMA microcellular foams. Polymer, 2005;46:11896-11903.

[12] Varghese AG, Batra RC. Constitutive equations for thrmomechanical deformations of glassy polymers. International Journal of Solids \& Structures, 2009;46:4079-4094.

[13] Cheng G, Gelin JC, Barriere T. Physical modelling and identification of polymer viscoelastic behaviour above glass transition temperature and application to the numerical simulation of the hot embossing process. Key Engineering Materials, 2013;554-557:1763-1776.

[14] Cheng G, Sahli M, Gelin JC, Barriere T. Physical modelling, numerical simulation and experimental investigation of microfluidic devices with amorphous thermoplastic polymers using a hot embossing process. Journal of Materials Processing Technology, 2016;229:36-53.

[15] Chang JR, Lin WJ, Huang CJ, Choi ST. Vibration and stability of an axially moving Rayleigh beam. Applied Mathematical Modelling, 2010;34(6):1482-1497. 
[16] Permoon MR, Rashidinia J, Parsa A, Haddadpour H, Salehi R. Application of radial basis functions and sinc method for solving the forced vibration of fractional viscoelastic beam. Journal of Mechanical Science and Technology, 2016;30(7):3001-3008.

[17] Demir DD, Bildik N, Sinir BG. Linear dynamical analysis of fractionally damped beams and rods. Journal of Engineering Mathematics, 2014;85(1):131-147.

[18] Akinyemi L, Iyiola OS. Exact and approximate solutions of timefractional models arising from physics via Shehu transform. Mathematical Methods in the Applied Sciences, 2020;43(12):7442-7464.

[19] Şenol M, Iyiola OS, Kasmaei HD, Lanre A. Efficient analytical techniques for solving time-fractional nonlinear coupled Jaulent-Miodek system with energy-dependent Schrödinger potential. Advances in Difference Equations, 2019;2019(1):1-21.

[20] Akinyemi L. q-Homotopy analysis method for solving the seventh-order time-fractional Lax's Korteweg-de Vries and Sawada-Kotera equations. Computational \& Applied Mathematics, 2019;38(4):1-22.

370 [21] Akinyemi L, Iyiola OS, Akpan U. Iterative methods for solving fourth and sixth order time-fractional Cahn-Hillard equation. Mathematical Methods in the Applied Sciences, 2020;43(7):4050-4074.

[22] Al-Raeei M, El-Daher MS. Numerical simulation of the space dependent fractional Schrdinger equation for London dispersion potential type. $375 \quad$ Heliyon, 2020;6(7):e04495.

[23] Wang J, Xu TZ, Wei YQ, Xie JQ. Numerical solutions for systems of fractional order differential equations with Bernoulli wavelets. International Journal of Computer Mathematics, 2018;96(2):317-336.

[24] Meng ZJ, Yi MX, Huang J, Lei S. Numerical solutions of nonlinear fractional differential equations by alternative Legendre polynomials. Applied Mathematics and Computation, 2018;336:454-464.

[25] Chen YM, Liu LQ, Liu DY, Boutat D. Numerical study of a class of variable order nonlinear fractional differential equation in terms of 
Bernstein polynomials. Ain Shams Engineering Journal, 2018;9(4):12351241.

[26] Chen YM, Liu LQ, Li BF, Sun YN. Numerical solution for the variable order linear cable equation with Bernstein polynomials. Applied Mathematics and Computation, 2014;238(7):329-341.

[27] Xie JQ, Yao ZB, Gui HL, Zhao FQ, Li DY. A two-dimensional Chebyshev wavelets approach for solving the Fokker-Planck equations of time and space fractional derivatives type with variable coefficients. Applied Mathematics and Computation, 2018;332:197-208.

[28] Chen YM, Sun L, Li X, Fu XH. Numerical solution of nonlinear fractional integral differential equations by using the second kind Chebyshev 395 wavelets. Computer Modeling in Engineering and Sciences, 2013;90(5):359378.

[29] Chen YM, Sun YN, Liu LQ. Numerical solution of fractional partial differential equations with variable coefficients using generalized fractionalorder Legendre functions. Applied Mathematics and Computation, 2014;244:847-858.

[30] Yi MX, Huang J. Wavelet operational matrix method for solvig fractional differential equations with variable coefficients. Applied Mathematics and Computation, 2014;230:383-394.

[31]. Wilhelm F. Viscoelasticity. New York: Springer-Verlag Berlin Heidelberg GmbH, 1975.

[32] Shen M, Chen S, Liu F. Unsteady MHD flow and heat transfer of fractional Maxwell viscoelastic nanofluid with Cattaneo heat flux and different particle shapes. Chinese Journal of Physics, 2018;56(3):1199-1211.

[33] Xu HY, Jiang XY. Creep constitutive models for viscoelastic materials 410 based on fractional derivatives. Computers and mathematics with Applications, 2017;73:1377-1384.

[34] Mokhtari M, Permoon MR, Haddadpour H. Aeroelastic analysis of sandwich cylinder with fractional viscoelastic core described by Zener model. Journal of Fluids and Structures, 2019;85:1-16. 
[35] Jinkyu K, Gary FD, Young KJ. Extended framework of Hamilton's principle for continuum dynamics. International Journal of Solids \& Structures, 2013;50(20-21):3418-3429.

[36] Wang L, Chen YM. Shifted-Chebyshev-polynomial-based numerical algorithm for fractional order polymer visco-elastic rotating beam. Chaos

$420 \quad$ Solitons \& Fractals, 2020;132:109585.

[37] Meng ZJ, Yi MX, Huang J, Song L. Numerical solutions of nonlinear fractional differential equations by alternative Legendre polynomials. Applied Mathematics and Computation, 2018;336:454-464.

\section{Appendix A. Symbol description}

\begin{tabular}{ll}
\hline symbol & explanation \\
\hline$D_{x}^{\alpha}$ & Caputo fractional derivative operator \\
$C, \lambda$ & constant \\
$x$ & position \\
$t$ & time \\
$p_{r}, q_{r}$ & traditional constitutive model parameter \\
$p_{\alpha_{r}}, q_{\alpha_{r}}$ & traditional fractional constitutive model parameter \\
$q_{\alpha}, q_{0}, q_{1}, \alpha$ & improved fractional constitutive model parameter \\
$\sigma(t)$ & stress \\
$\varepsilon(t)$ & strain \\
$\rho$ & material density \\
$W$ & work done by external forces \\
$y(x, t)$ & displacement \\
$E^{*}$ & complex elastic modulus \\
$E^{\prime}$ & storage modulus \\
$E^{\prime \prime}$ & loss modulus \\
$A_{x}$ & cross-sectional area \\
$E_{k}$ & kinetic energy \\
$V$ & potential energy \\
$T$ & temperature \\
$I$ & moment of inertia \\
$f(x, t)$ & external load \\
$H$ & length \\
$n$ & number of terms of the shifted Legendre polynomial
\end{tabular}


$l_{n, i}(x), L_{n, i}(x), \bar{L}_{n, i}(t) \quad$ shifted Legendre ploynomial

$A, C, K, U, E, F, L, M, \Delta, \delta \quad$ coefficient matrix

$\varphi_{n}, \Phi_{n}, \phi_{n}$

$T_{n}$

$\langle$,

family of shifted Legendre ploynomials

$u_{n}$

family of basical ploynomials

$D_{x}$

Inner product

$n$ exponent th functional approximation

$D_{m x}$

first order differential operator matrix for $x$

$D_{t}$

$m$ exponent th differential operator matrix for $x$

$D_{v t}$

first order differential operator matrix for $t$

$v$ exponent th differential operator matrix for $t$

$D_{\alpha t}$ $\alpha$ exponent th differential operator matrix for $t$ 


\section{Declaration of interests}

$\checkmark$ The authors declare that they have no known competing financial interests or personal relationships that could have appeared to influence the work reported in this paper.

$\square$ The authors declare the following financial interests/personal relationships which may be considered as potential competing interests:

There are no conflicts of interest. 
Author Contributions: Cao Jiawei wrote some sections of the manuscript and performed analyses; Yiming Chen, Wang Yuanhui, Gang Cheng and Thierry Barrière prepared some other sections of the paper. All authors read and approved the final manuscript. 